\title{
Nanoparticle ligands and pyrolized graphitic carbon in CZTSSe photovoltaic devices
}

\section{Supporting Information}

Trevor R. Martin, ${ }^{1,4}$ John K. Katahara, ${ }^{2,4}$ Cori N. Bucherl, ${ }^{2,4}$ Benjamin W. Krueger, ${ }^{3}$ Hugh W. Hillhouse $^{2,4^{*}}$ and Christine K. Luscombe $\mathrm{e}^{1,4,5^{* *}}$

${ }^{1}$ Department of Materials Science and Engineering, University of Washington, Seattle, WA 98195-2120, ${ }^{2}$ Department of Chemical Engineering, University of Washington, Seattle, WA 98195-1750, ${ }^{3}$ Department of Physics, University of Washington, Seattle, WA 98195-1560, ${ }^{4}$ Molecular Engineering and Sciences Institute, University of Washington, Seattle, WA 98195$1652,{ }^{5}$ Department of Chemistry, University of Washington, Seattle, WA 98195-1700

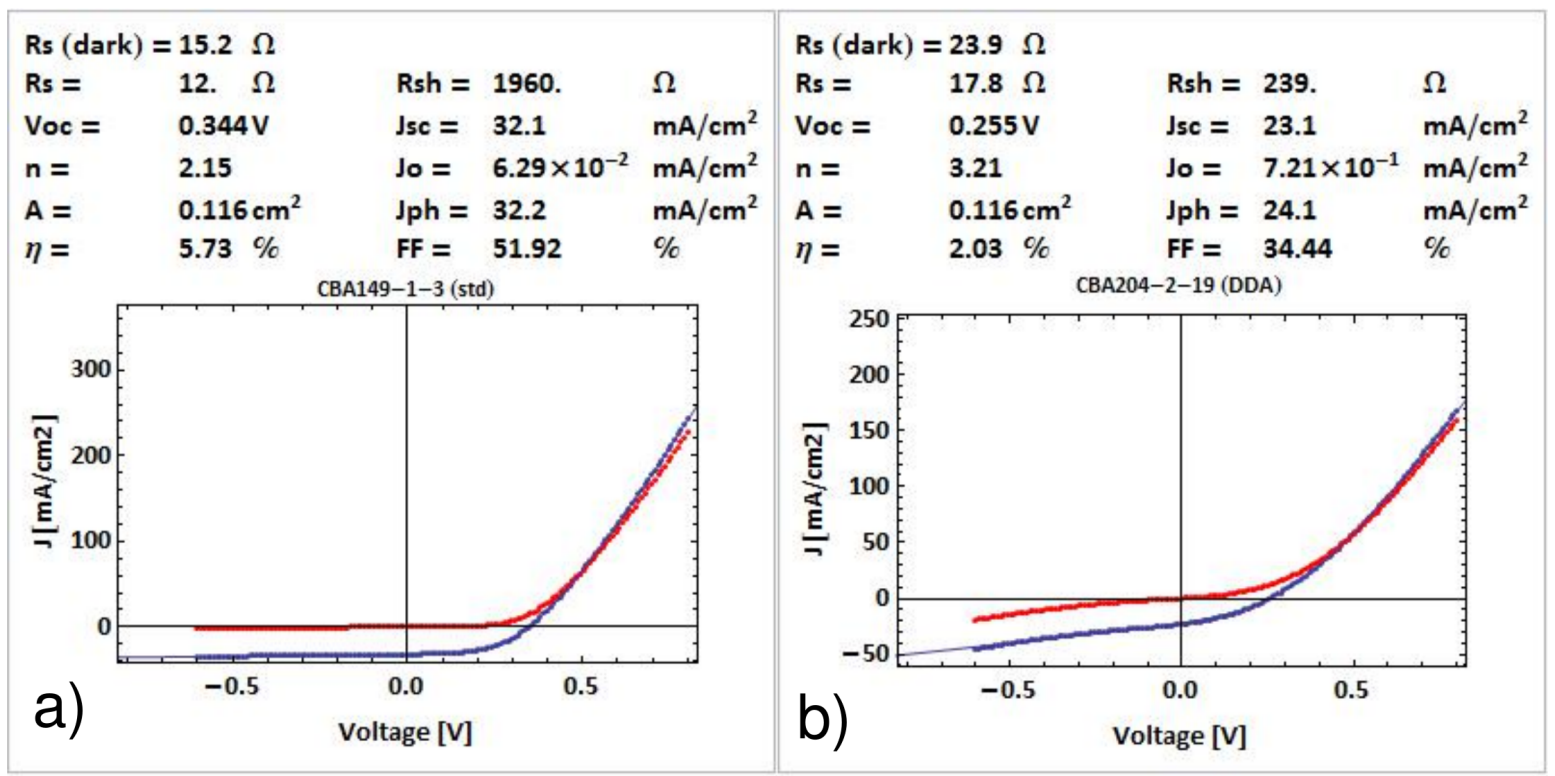

Figure S1: JV curves and the corresponding performance data for representative a) OLA-based and b) DDA-based CZTSSe photovoltaic devices. 

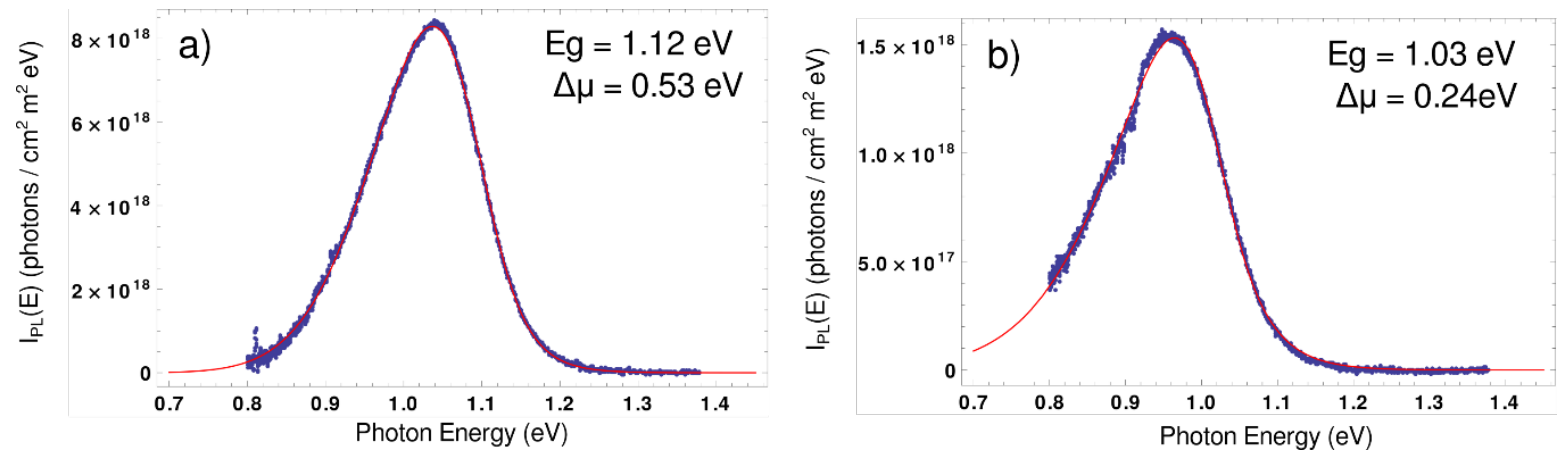

Figure S2: PL data with curve fit for a) OLA-based and b) DDA-based CZTSSe devices

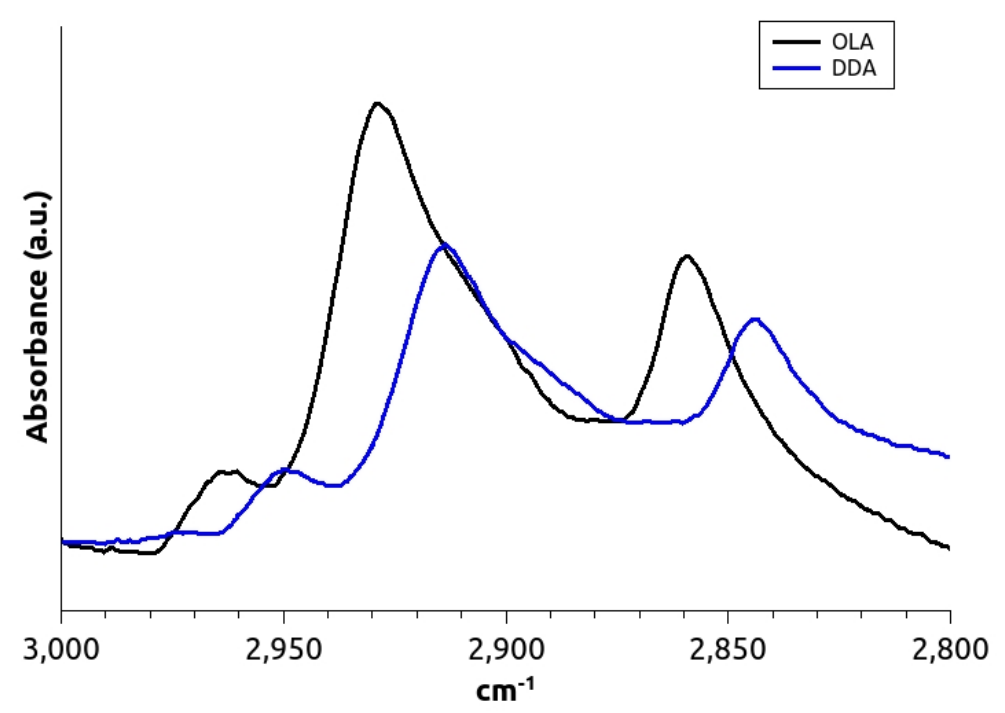

Figure S3: FTIR of pre-annealed CZTS nanoparticle films showing C-H resonance peaks, which indicates the presence of remaining aliphatic ligands prior to the annealing step.

\section{XPS Analysis}

Annealed CZTS nanoparticle films were mounted into the analysis chamber and Survey, $\mathrm{C} 1 \mathrm{~s}, \mathrm{~S} 2 \mathrm{p} / \mathrm{Se} 3 \mathrm{p}$, and Se 3d spectra were obtained. Figures S4a and S4b show S 2p/Se 3p spectra after annealing for each ligand. Each of the spectra were fit using Voigt peaks according to the following strategy. Se $3 p$ and S 2p overlap, and Se 3s and S 2s overlap, but no S spectra coincide with Se 3d, which provides a way to isolate the Se contribution to the combined Se $3 p / S 2 p$ spectrum. Figures S4c and S4d show Se 3d spectra after annealing for each film. In each spectrum three main components are visible at approximately $54 \mathrm{eV}, 56 \mathrm{eV}$, and $59 \mathrm{eV}$, and are attributed to the Se 2-, Se 0+ and Se 4+ oxidation states, respectively. For each peak a spin-orbit splitting of $0.86 \mathrm{eV}$ was used, and the Lorentzian and Gaussian widths were constrained to be the same for all component peaks.

When comparing Se 3d to Se 3p (and Se 3s) spectra, components arising from different bonding states should appear at roughly the same binding energy separation and in roughly the same relative intensities. The spin-orbit split for $\mathrm{Se} 3 \mathrm{p}$ is $5.8 \mathrm{eV}$, the spacing between components and relative amplitudes are constrained to match the corresponding Se $3 \mathrm{~d}$ spectrum, 
and the Lorentzian and Gaussian widths are each held to be uniform. S $2 p$ components are expected to appear at roughly the same binding energies as in the before-annealed spectra, namely at $161 \mathrm{eV}$ and $163 \mathrm{eV}$ for 2- and 0+, respectively, with a spin-orbit splitting of $1.18 \mathrm{eV}$. Fixing the S $2 p$ peak positions but allowing their amplitudes to vary, constraining the $S 2 p$ Gaussian widths (attributed to surface non-uniformity and equipment broadening) to match the fit value for Se $3 p$ components, but allowing the S $2 p$ Lorentzian widths (determined by the unique photoelectron lifetime for each element) to vary, produced the published result. Despite the large number of restrictions imposed due to physical considerations, the agreement between fit and data is remarkable.

a)

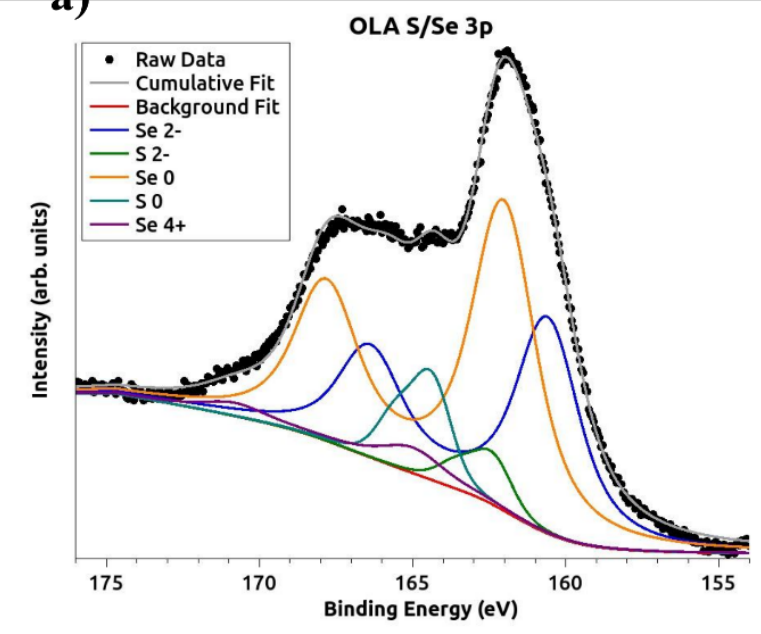

b)

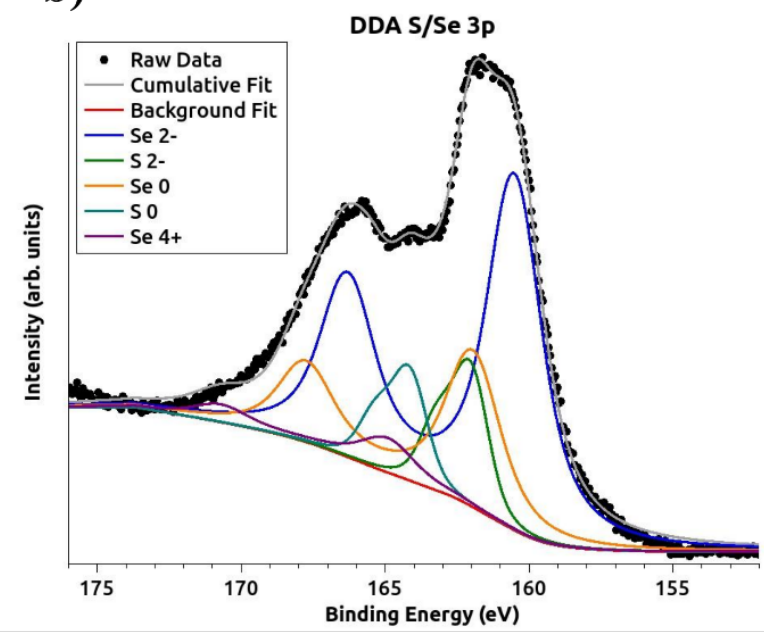

c)

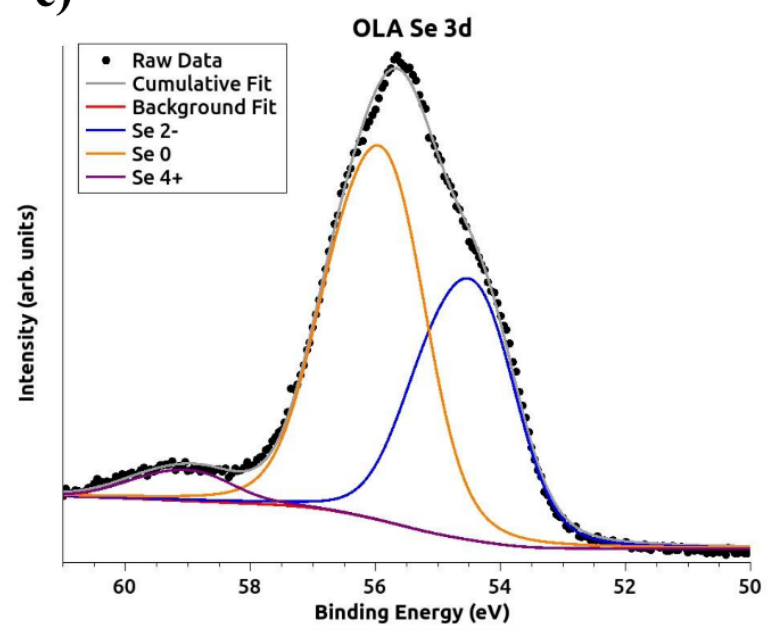

d)

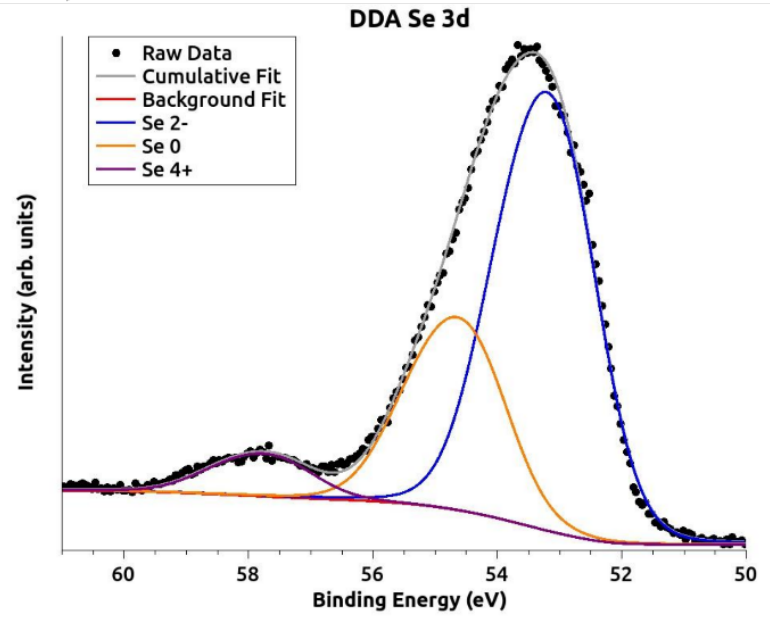

Figure S4: Fitted XPS data for annealed OLA (a and c) and DDA (b and d) CZTSSe films. 


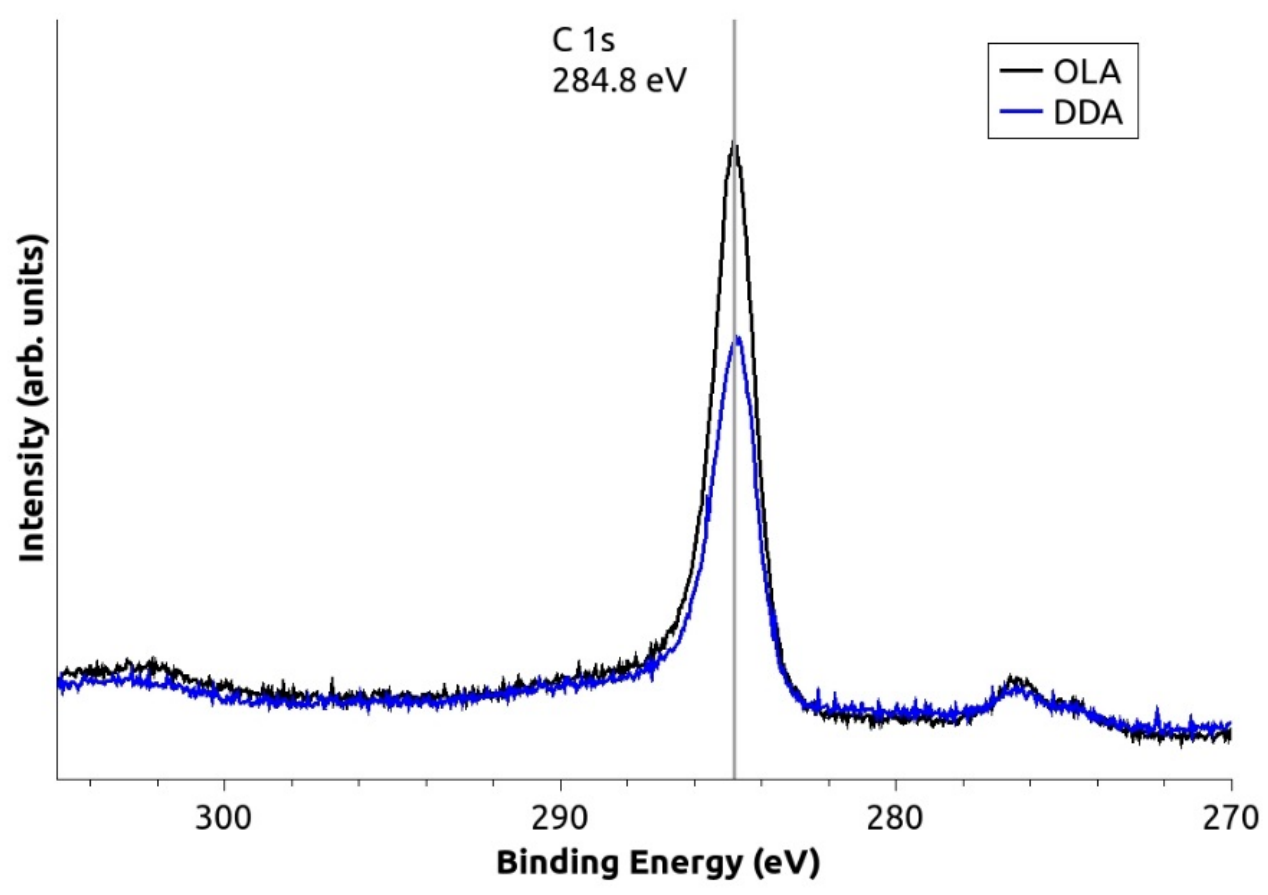

Figure S5: XPS C 1s data for annealed CZTSSe films.

\section{Electrical Conductivity of Graphitic Carbon Films}

Four-point probe measurements as well as profilometry measurements were used to calculate the conductivity of the carbon thin films using the standard thin film conductivity equation presented in Equation S1, where $d$ is the thickness of the film and $R$ is the resistance.

$\sigma=\frac{\ln (2)}{\pi d R} \quad$ (Equation $\left.\mathrm{S} 1\right)$

Despite the significant uncertainty present in the data due to a large variation in film thicknesses, the measurements in Table S1 can be used to estimate the electrical conductivity of the films.

Table S1: Conductivity of Carbon Films

\begin{tabular}{|l|l|l|l|}
\hline Ligands & $\begin{array}{l}\text { Average } \\
\text { Thickness } \\
(\mathrm{nm})\end{array}$ & $\begin{array}{l}\text { Average } \\
\text { Resistance } \\
(\mathrm{M} \Omega)\end{array}$ & $\begin{array}{l}\text { Conductance } \\
\left(\mathrm{S} / \mathrm{cm}^{2}\right)\end{array}$ \\
\hline OLA & $175 \pm 60$ & $20.4 \pm 0.3$ & $(6.1 \pm 2.2) \times 10^{-4}$ \\
\hline DDA & $130 \pm 100$ & $15.1 \pm 5.4$ & $(11.1 \pm 9.6) \times 10^{-4}$ \\
\hline
\end{tabular}

